\title{
Immune Checkpoint Inhibitors Therapy in Prostate Cancer: Lessons Learnt
}

\section{Prabhakar S and Chandok M*}

Ambay Immune Sensors and Controls, LLC, USA

*Corresponding author: Meena Chandok, Ambay Immune Sensors and Controls, LLC Suite 118, Technical Innovation Center, 20140 Scholar Drive, Hagerstown, MD-21742, USA, Email: ambayservices@gmail.com

\section{Review Article}

Volume 2 Issue 1

Received Date: May 18, 2020

Published Date: June 26, 2020

DOI: $10.23880 /$ aii-16000119

\section{Abstract}

Immune checkpoint inhibitors (ICIs) therapy has rapidly changed the treatment landscape for a number of cancers but resulted in only marginal success in prostate cancer (PCa). In order to bring about durable clinical benefit of immune check point inhibitor therapy in prostate cancer, it is critical to understand the current status of ICIs therapy for PCa and reasons for low/negligible outcomes. This article summarizes the current status of ICIs therapy in PCa and discusses how ICIs therapy can result in the successful treatment of a specific subset of PCa patients. In this article, we review how immunotherapy and different combination therapies are paving their way by incorporating the strategies of converting the "cold" PCa tumor into "hot", role of genomic landscape of PCa in influencing the outcome of ICIs therapy, what are the promising novel biomarker candidates, which can guide the patient selection for ICIs therapy and help in monitoring the efficacy of treatment. This article highlights that ICIs therapy hold a promising future in subset of PCa patients.

Keywords: Immune Checkpoint Inhibitors (ICIs); Prostate Cancer (PCa); Metastatic Castration-Resistant Prostate Cancer (mCRPC)

Abbreviations: ICIs: Immune Checkpoint Inhibitors; PCa: Prostate Cancer; ADT: Androgen Deprivation Therapy; mCRPC: Metastatic Castration-Resistant Prostate Cancer; DCR: Disease Control Rate; ATM: Ataxia-Telangiesctasia Mutated; DDR: DNA Damage Repair; HRD: Homologous Recombination Deficiency; MANAs: Mutation-Associated Neoantigens; NSCLC: Non-Small-Cell Lung Cancer; HR: Homologous Recombination; DMMR: DNA Mismatch Repair; TIME: Tumor Immune Microenvironment; TAMs: TumorAssociated Macrophages; RT: Radiation Therapy; OS: Overall Survival; BRD4: Bromodomain-Containing Protein 4; EZH2: Enhancer of Zeste Homolog-2; PARP: Poly ADP-Ribose Polymerase; IFN: Interferon.

\section{Introduction}

Prostate cancer (PCa) is the second most commonly diagnosed malignant tumor in men, and a major cause of mortality, with more than a million new cases and 359,000 deaths world-wide, in 2018 [1]. The range of currently prevalent treatment options for PCa (surgery, radiation, androgen deprivation and chemotherapy) pose adverse effects and show very limited efficacy for metastatic and treatment resistant disease [2]. PCa patients respond to androgen deprivation therapy (ADT) initially but almost all patients progress to metastatic castration-resistant prostate cancer (mCRPC) [3]. Food and Drug Administration (FDA) approved chemotherapy treatments for MCRPC, docetaxel and cabazitaxel, in combination with enzalutamide, abiraterone and Sipuleucel-T (Sip-T dendritic cell vaccine) [4-9], only increase median survival benefit by $2-4$ months $[10,11]$. Other therapies are therefore being explored to treat mCRPC patients, and one of the most promising ones is immunotherapy with immune check point inhibitors 
(ICIs). Clinical data suggests that $5-12 \%$ of mCRPC patients benefit from immune check point blockade $[12,13]$. Immune checkpoint inhibitors are antibodies that target regulatory or co-inhibitory signaling molecules, including cytotoxic T-lymphocyte antigen-4 (CTLA-4), programmed cell death-1 (PD-1), and programmed cell death ligand-1 (PD-L1). These three proteins are called immune check points, since overexpression of these proteins by tumor cells or T cells is one of the mechanisms that tumor cells employ to evade $\mathrm{T}$ cell mediated attack, enabling proliferation of tumor cells. Blocking these markers leads to activation of $\mathrm{T}$ cell and antitumor response [14]. PD-1/PD-L1 inhibition is one of the most promising immunotherapies across many different refractory cancers including melanoma, non-small-cell lung carcinoma, and renal cell carcinomas [15-16] and yet the effectivity of this approach in many cancers including PCa is very limited $[17,18]$. In this article, we focus on the current status of ICIs therapy for PCa, reasons for low/negligible objective responses, ways to make ICIs therapy a viable option with durable clinical benefit in PCa and the associated challenges to achieve this goal.

\section{Monotherapy with ICIs in PCa}

Ipilimumab, an antibody against CTLA-4, known to block the inhibition of $\mathrm{T}$ cell response and allow immune cells to recognize and kill tumor cells, was approved by FDA in 2011 to treat metastatic melanoma [19]. Randomized Phase III clinical trials with Ipilimumab in asymptomatic or minimally symptomatic patients with metastatic chemotherapy-naïve castration-resistant prostate cancer showed some clinical antitumor activity but subsequent Phase III trials failed to show significant difference in overall survival $[18,20]$. Therapies targeting the PD-1, Nivolumab also did not result in significant objective clinical responses for PCa patients [16,21]. In spite of these early disappointing results, the enthusiasm to investigate ICIs in PCa remains high and the monotherapy clinical trials using anti-PD1 antibody, Pembrolizumab in mCRPC for PD-L1 positive, PDL1 negative and bone metastasis/non-measurable disease (NCT02787005) are still active. The currently available results from these clinical trials indicate that the median overall survival ranged from 7.9 to 14.1 months, and that the disease control rate (DCR) are 10\%, 9\% and 22\% in PD-L1-positive, PD-L1-negative, and bone-predominant non-measurable disease patients, respectively [22]. Though these results indicate only modest antitumor activity, further analysis displayed marginal increase in response with patients harboring mutations in breast cancer gene (BRCA1/2) or Ataxia-Telangiesctasia mutated (ATM) gene (objective response rate (ORR) 11\%) [22]. Similarly, other clinical trial results $[23,24]$ also suggest that monotherapy with PD-1/PD-L1 ICIs is not enough to deliver significant responses.

\section{Combination Therapy with ICIs in PCa}

Despite the low success rate of PD-1/PD-L1 therapy in PCa, PD-1/PD-L1 axis remains an area of therapeutic interest in advanced PCa as the expression of PD-L1 is up regulated in mCRPC4, and PD-L1 expression increases with other treatments [25-27], drawing attention to the PD-L1 blockade in combination with other therapeutic modalities. A CRPC preclinical study, using radiotherapy in combination with anti-PD-1 and anti-PD-L1 increased the median survival rates to $70 \%$ and $130 \%$, respectively, compared to the drug alone, demonstrating that robust responses are achievable in PCa by combining anti-PD-1/PD-L1 blockade therapy with other modalities [28]. Several clinical trials using PD-1/PD-L1 antibodies with different therapies/vaccines like PROSTVAC (NCT02933255), pTVG-HP (NCT03600350), chemotherapy (NCT03572478, NCT03170960, NCT03673787), radium-223 (NCT03093428, NCT02814669), Sip-T (NCT03024216), and CTLA-4 checkpoint inhibitors (NCT03333616) are in progress to assess the efficacy of ICIs combination therapy for PCa. A clinical trial (Phase Ib/II) for mCRPC patients, involving Pembrolizumab with anti-hormonal therapy, docetaxel, or targeted therapy with poly-ADP ribose polymerase (PARP) inhibitor (KEYNOTE-365) is currently active and showing promising outcomes. In another phase II study, where 10 patients were treated with enzalutamide (anti-androgen drug) and Pembrolizumab (anti-PD-1 antibody), 5 patients showed reduction in PSA levels and tumor size [12].

The anti-CTLA-4 antibody, Ipilimumab, is also being tested in clinical trials with radiation (NCT03477864), Nivolumab (anti-PD-1 antibody) [NCT03333616, NCT03061539, NCT02985957 (Checkmate 650)], chemotherapy (NCT03098160, NCT01688492), and AST (NCT01498978).

The combination of Nivolumab and Ipilimumab for mCRPC patients (Checkmate650) displayed impressive clinical responses in patients who showed PD-L1-positivity, DNA damage repair (DDR) mutations, homologous recombination deficiency (HRD), and high tumor mutational burden (TMB). The outcome of this study has generated enthusiasm, to explore the role of genomic landscape to improve clinical outcomes in PCa patients, using ICIs therapy.

\section{Genomic Landscape of PCa and ICIs}

Large-scale genomic analysis identifying specific genetic mutations will enhance our understanding, and how genomic characteristics of tumor can shape the future of ICIs therapy in PCa. It is known that tumors that have high somatic mutational load, especially nonsynonymous alterations, lead to more mutation-associated neoantigens (MANAs) that are recognized by the T cells which attack the 
tumor [29]. PD-1 therapy has proved to be successful in both Melanoma [19,30] and Non-Small-Cell Lung Cancer (NSCLC) cancers $[31,32]$ which show a high mutational load. PCa is generally not considered a cancer with high mutational load since on average, $\mathrm{PCa}$ has between 50-100 nonsynonymous DNA alterations per cancer exome (i.e. 1-2 mutations per $\mathrm{Mb}$ ) [33]. A comprehensive multi-institutional study of mCRPC tumors done by Robinson, et al. revealed that 8-12\% of patients harbor either germ line mutations or $20-25 \%$ acquires somatic mutations in genes involved in homologous recombination (HR) repair [34]. The association of HR mutations, including mismatch repair (MMR) mutations with high PD-L1 expression and increase in T cell infiltration, makes ICIs therapy a very relevant option for HR-deficient advanced PCa patients [35]. A study by Pritchar, et al. [36], indicates that $5-12 \%$ of advanced PCa patients may be hypermutated due to MMR gene mutations and Microsatellite Instability-High (MSI-H) phenotypes [37]. This subgroup of patients may benefit from Pembrolizumab, an FDA approved drug for treatment of solid tumors with DNA mismatch repair (dMMR) mutations.

A comprehensive analysis of genomics, transcriptomics, and clinical data from 124 mCRPC patients allowed the identification of two distinct dMMR-associated mutational signatures that are prevalent in advanced PCa [38]. These mutational signatures were also associated with higher immune cell infiltration (including subsets of T cells, NK cells, and myeloid cells), increased expression of $\mathrm{T}$ cell related transcripts, and PD-L1 and PD-L2 expression, indicating that in some dMMR metastatic CRPC patients, the efficacy of ICIs can be enhanced via developing different combination strategies aimed at depleting the myeloid subsets in tumor.

Another interesting report identified a novel genetic subtype of $\mathrm{PCa}$, where mutations in the transcriptionregulating gene CDK12 [39] were associated with a very high neoantigen burden and increased infiltration of $\mathrm{T}$ cells, recommending this subset of PCa patients as a good candidate for ICIs therapy. A phase II trial of 17 mCRPC patients treated with PD-L1 inhibitor, Durvalumab, and Olaparib, a PARP inhibitor, indicated that 35\% (6/17) who harbored DNA Damage Repair (DDR) mutation (all at BRCA2 lesions) responded better to the combination therapy [40]. The trial is now expanding to recruit 50 mCRPC patients.

Study from Abida, et al. [41] using tumor and germline sequencing for $1033 \mathrm{PCa}$ patients demonstrated that 3.1\% of the patients show MSI or dMMR characteristic [41]. Out of 11 patients, 4 patients showed radiographic response, after undergoing PD-1/PD-L1 therapy. They also noticed that $21.9 \%$ of the patients had germ line mutations and the rest of them acquired somatic mutations during the disease.
In addition to expression of immune check point molecules and genomic landscape of $\mathrm{PCa}$, the success of ICIs therapy is governed by the type of tumor immune microenvironment (TIME) present in the tumor.

\section{Tumor Immune Microenvironment (TIME): Hindrances to ICIs Therapy}

One of the reasons for very limited success of PCa to ICIs, is the presence of antitumor immune suppressive TIME which leads to paucity of immune cell infiltration (immunological "cold" microenvironment) [42,43]. Combinations therapies with ICIs which can change the "cold" PCa TIME to immunologically "hot" by decreasing the immune suppression and driving the T-cells back to the tumor, will bring the long-waited breakthrough for use of ICIs in PCa. The current evidences show that TIME of PCa mainly consists of a sub-population of Tregs and pro-tumorigenic M2-subtype, tumor-associated macrophages (TAMs). TAMs secrete high levels of TGF- $\beta$ and create an immune suppressive environment in a variety of ways $[44,45]$. Presence of TGF- $\beta$ has been shown to favor immune exclusion by influencing the environment towards poor infiltration of TIL [46]. T-regs lead to dampening of the immune response and produce inhibitory cytokines to maintain self-tolerance $[47,48]$. The presence of chemokines in TIME further contributes to orchestrating the responses towards immune suppression [49-53]. Clinical trials of therapies/agents which reprogram the TIME, leading to immune infiltration, activation of T-cells and enhancing tumor immunity, along with ICIs to remove the breaks, will provide stable clinical benefits in PCa. Developing new non-invasive methods to monitor the efficacy of employed combination therapies will provide insights and explain why some combination therapies are unable to deliver the desired outcomes and how to overcome these barriers.

\section{Converting "Cold Tumor" to "Hot Tumor": Combination Therapy with ICIs}

Conventional Therapies: Radiation therapy (RT) alters the TIME by leading to immunogenic cell death, inducing inflammatory cytokines, recruiting dendritic cells and activating tumor-specific $T$ cells [54]. The induction of a potent immune response at the sites of irradiated disease, as well as at distant locations ("abscopal effect") [55,56] makes combination of ICIs with RT an attractive option. Since the immunomodulatory properties of RT depend on dose, fractionation, and site [57-60], incorporating these factors while designing the combination therapy trials will be important for successful outcomes of combination therapy with ICIs. Current evidences from liver and lung cancer studies indicate the safety of the combination of RT with Ipilimumab however no overall survival (OS) benefit to most 
patients was noted [60]. The current efforts for RT with ICIs combination in PCa are listed in Table 1.

Chemotherapy in combination with ICIs is currently used as the standard of care in triple-negative breast cancer and lung cancer [61-64]. Cytotoxic chemotherapy with ICIs has been shown to reduce tumor burden, activate antigen cascade, and reduce MDSCs [65,66]. Chemotherapy with ICIs becomes an attractive option for a cancer like PCa which is sensitive to taxane, allowing docetaxel and paclitaxel to be investigated. The ongoing clinical trials of chemotherapy with ICIs are mentioned in Table 1.

\begin{tabular}{|c|c|}
\hline Therapies & Active Clinical Trials \\
\hline Radiotherapy & NCT03543189, NCT03795207,NCT03217747,NCT01303705 \\
\hline Chemotherapy & NCT03951831, NCT03879122, NCT03248570, NCT03834506 \\
\hline TGF- $\beta$ & NCT03685591, NCT02452008, \\
\hline IL-8 & NCT03689699 \\
\hline Adenosine pathway & NCT03454451, NCT03629756, NCT04089553 \\
\hline Androgen Deprivation Therapy & NCT03016312, NCT03753243, NCT03543189 \\
\hline PARP Inhibitors & NCT03572478, NCT03330405, NCT02484404, NCT03810105 \\
\hline
\end{tabular}

Table 1: Active Clinical Trials for Combination Therapy with ICIs.

Epigenetics Factors: Epigenetics factors, like p300, bromodomain-containing protein 4 (BRD4) and the enhancer of zeste homolog-2 (EZH2), have shown promising outcomes in PCa treatment [67-69]. PD-L1 correlated with p300 has been involved in progression of $\mathrm{PCa}$ [70] and targeting of p300, by p300/CBP inhibitor, A485, combined with anti-PD-L1 antibody, reactivates T-cells function towards anti-tumor immunity, suggesting that the combination of epigenetic factor inhibitor with ICIs enhances the efficacy of ICIs in PCa [70].

EZH2 is involved in chronic inflammation and tumor immune tolerance [71] in the TIME. EZH2 is over-expressed in prostate cancer and is known to negatively regulate IFN response genes [72], affect antigen presentation, Th-1 chemokine signaling [73], and PD-L1 [74]. EZH2 inhibition, combined with PD-1, significantly enhanced anti-tumor response and reprogrammed the TIME by significantly increasing the intra-tumoral trafficking of activated CD8+ T-cells and M-subtype TAMs with concurrent loss of M2subtype TAMs. Interestingly, monotherapy of EZH2 inhibition or PD-1 fail to display a similar outcome, indicating that the inhibition of EZH2 has the potential to enhance PCa response to PD-1, ICI therapy.

Cytokines: TGF- $\beta$ present in TIME contributes to the immune suppressive environment and mediates immune resistance in PCa [75]. Hence, altering the level of TGF- $\beta$ in TIME could offer ways to overcome the barriers in the success of ICIs therapy. Evidence indicates that the combination therapy of ICIs with an anti-TGF- $\beta$ antibody induced changes in TIME environment and polarized the CD $4+\mathrm{T}$ cells to the Th- 1 cell subset with increase in expansion of CD8+ effector memory cells to control tumor growth [76]. Clinical trials investigating the role of TGF- $\beta$ in combination therapy are listed in Table 1.

IL-8, a proinflammatory cytokine modulated by androgens, has been shown to increase the infiltration of MDSCs [77] and a clinical trial for the use of an IL-8 antibody in combination with Nivolumab and ADT are underway (Table 1).

Adenosine Pathway: Adenosine signaling has evolved as a powerful immuno-metabolic checkpoint in tumors, to target the inhibitory mechanisms in the TIME and reprogram it towards anti-tumor immunity [78]. Initial results of a phase I trial evaluating the A2A receptor inhibitor AZD4653 (which blocks the adenosine from its receptor), alone or in combination with the anti-PD-L1 antibody Durvalumab, showed a response rate of $37.5 \%$ in $\mathrm{mCRPC}$ patients, with a durable PSA decline greater than $99 \%$ in $25 \%$ of the patients [79]. Further trials are underway to explore this pathway in PCa patients (Table 1).

Androgen Deprivation Therapy (ADT): ADT is currently used among the first line therapies for mCRPC. Growing evidence indicates that androgens and androgen deprivation have profound effects on the immune system [80], besides affecting prostate tumor cells directly. The interest in use of ADT to modulate tumor cell sensitivity to T-cells and increase the infiltration of T-cell into the prostate [81] is therefore enormous. Clinical trials are currently investigating combinations of ADT and ICIs in MCRPC patients (Table 1).

Poly ADP - Ribose Polymerase (PARP) Inhibitors: Recent evidence show that PARP inhibition can alleviate the resistance and enhance the efficacy of ICIs therapy by promoting cross-presentation and modifying TIME [82-84]. 
The preliminary results from clinical trial (NCT02484404) indicate that the combination therapy of Olaparib and Durvalumab effectively reduced the tumor burden (measured by PSA reduction $>50 \%$ ) in 8/17 unselected mCRPC patients [85]. Mutation in DNA damage response (DDR) emerged as a favorable biomarker, to indicate the outcome of the combination therapy (12-month progression-free survival probability of deficient DDR vs. proficient DDR, $83.3 \%$ vs. $36.4 \%, \mathrm{P}=0.03$ ) [85,86]. Additional trials evaluating the efficacy of ICIs and PARP inhibitors combination in mCRPC patients are underway (Table 1).

It is becoming apparent that ICIs therapy in $\mathrm{PCa}$ can produce a durable response in subsets of patients. Identification of the subsets of patients who can show favorable/durable response to ICIs therapy is the major challenge. Therefore, the immediate need is to develop reliable biomarkers which can guide patient selection and allow monitoring the efficacy of ICIs therapy.

\section{Predictive Biomarkers for Patient Selection and Response to ICIs Therapy: An Existing Challenge}

Currently, most clinical trials do not employ upfront stratification/selection, to enrich the sensitive patient populations. The clinical trial design based on biomarker(s)based patient-enrichment strategies will offer multi-faceted benefits, like avoiding toxic side effects for patients who do not respond, saving cost, improving the quality of life and better outcome/response rate.

Considering the tumor immune contexture and molecular landscape of $\mathrm{PCa}$, it appears that while single biomarker may pose substantial limitations, a combination of biomarkers which can reveal the interaction of host and tumor could enable a precision medicine approach in ICIs therapy. Potential biomarkers capable of selecting the patients and guiding the ICIs therapy in PCa are discussed below.

PD-L1 Expression: The overexpression of PD-L1 is generally associated with the response rate to ICIs therapy, and whether this holds true in case of $\mathrm{PCa}$, requires further investigation. Using expression of PD-L1 in tumor cells, the initial clinical trial (KEYNOTE-028) insinuated that PD-L1 expression could predict response to ICIs $[87,88]$, but the larger trial (KEYNOTE-199) revealed no change in response rates between the PD-L1-and PD-L1+ cohorts [89], suggesting that PD-L1 may not be a prefect predictive biomarker. Lack of standardization makes this true even in malignancies where it has been associated with clinical outcomes [90-93]. Due to usage of different criteria and assays employed across the trials, the debate is still on to define the parameter that constitutes positivity. Discrepancies in the results could also be attributed to significant inter-tumoral and intra-tumoral heterogeneity of PD-L1 expression, where one tumor sample may not provide adequate representation [94]. Studies also demonstrate that PD-L1 expression is inducible and its expression can change over the course of clinical treatment; hence the use of archival tissue for measuring static PD-L1 may not reflect the true status to guide patient selection and response to ICIs therapy.

Recent studies indicate that tumor cells release exosomes carrying PD-L1, which exert immunomodulatory effects [95], suppress T-cell function and antagonize anti-PD-1 response [96]. The comparison of tumor cell PD-L1 with exosomal PDL1 suggest that exosomal PD-L1 could be a better choice for being a predictive biomarker, as it reflects the state of the whole-body system and can predict the dynamic progress of the disease. The non-invasive methods of measuring the exosomal PD-L1 will allow testing at multiple time points, will overcome the problems related to tumor heterogeneity and offer differentiation of responders and non-responders during therapy. The changes in level of exosomal PD-L1 itself could be a strong indicator for evaluating the efficacy of antiPD-1 therapy. In PCa, the level of CD274, which encodes PDL1 mRNA, is higher than melanoma, which responds well to PD-L1 blockade. This indicates that most of the translated PD-L1 was secreted extracellularly by exosomes, which in fact could be inhibiting the T-cell function and contributing to the resistance to PD-L1 blockade treatment. Removal of exosomal PD-L1 allowed to overcome the resistance to PD-L1 blockade [70,97]. The results from other studies in melanoma and lung cancer [98] further corroborate this concept. All these evidences show strong potential for exosomal PD-L1 as a biomarker for improving the efficacy of ICIs therapy, which could provide appropriate information on clinical outcomes.

Since PCa actively suppresses antitumor immune responses by creating an immune-suppressive microenvironment, the identification of predictive biomarkers that would access the changes in the immune suppressive environment and predict a shift in anti-tumor immunity along with the status of exosomal PD-L1 would be most beneficial. We are currently focused on developing the biomarker(s) to assess the immune suppression status in PCa, which will aid in selecting the specific targeted population for ICIs combination therapy and assessing the outcome of therapy during treatment.

Genomic Biomarkers: As discussed above, tumor mutation burden (TMB), MSI-H/dMMR, CDK12 mutation and DDR defects show potential in identifying the subset of PCa patients who could generate favorable outcome upon ICIs therapy. In this section, we will explore the potential of these to be predictive reliable biomarkers for ICIs therapy. 
TMB is a promising biomarker which has been correlated with clinical outcomes in several malignancies $[87,99]$ and the efficacy of PD-1 inhibitors [100]. TMB in metastatic $\mathrm{PCa}$ is generally low and only 3 to 8 percent of advanced PCa show tumors with high TMB $[41,101]$. The possibility also exists that high TMB could be a genomic manifestation of dMMR [99]. Currently, there is no standardized cutoff for TMB, and ICIs responses are observed even with low TMB. Furthermore, detection of TMB requiring whole-exome sequencing, a highly complex and expensive process, makes this biomarker not very well suited for routine clinical use.

Patients with MSI-H or mismatch repair-deficient (dMMR) tumors are approved to receive ICI Pembrolizumab and MSI-H is believed to exist in approximately $3 \%$ of men with mCRPC [34,38,41,102]. Higher responsiveness of mismatch repair-deficient cancers to immune checkpoint inhibition, presumably due to the increased immunogenicity, which results from excessive DNA mutations [103], generated interest in this biomarker to guide the optimal ICIs therapy in the clinic.

$\mathrm{Wu}$ YM, et al. [39] proposed the possibility of biallelic somatic loss-of-function mutations in CDK12 as a biomarker for response to ICIs, as CDK12 alterations associated with focal tandem duplications led to gene fusions and generation of neoantigens. The results with alterations in CDK12 (approximately $6.9 \%$ of mCRPC patients) showing a high infiltration of T- cells suggested that mutation in CDK12 could allow selection of patients, who might have positive outcomes to ICIs therapy. Clinical trials (NCT03570619 and NCT03810105) to assess the efficacy of ICIs in patients with CDK12 alterations areunderway.

Tumor DDR defects are well known in determining the response to chemotherapeutics and RT. Up regulation of PDL1 expression in response to double-strand break damage in different cancer cell lines, including PCa [104] makes DDR an interesting candidate to be explored as a biomarker. In PCa, $22.7 \%$ of patients show DDR alterations (BRCA2 and ATM as most frequently affected) [34]. The outcome of a phase II trial where mCRPC patients responded better to the combination therapy that harbored DDR mutation, further establishes the association between tumor DDR status and ICIs response [40]. However, in order to utilize DDR to predict ICIs therapy response, parameters like sequencing depth, mutational frequency thresholds, and comparability of mutational definitions (genetic terminology) need to be defined clearly, so that the differences in the definitions of what is considered a mutation (monoallelic versus biallelic) should not hamper the progress [105].

\section{Conclusion}

As novel therapies are advancing, and rational combination therapies are emerging, the success of ICIs in PCa will soon bloom. The findings from different clinical trials indicate that the success of ICIs therapy is achievable in specific subsets of PCa patients with a durable response. The key elements which govern this success will require the use of therapies which allow reprogramming of TIME, reliable predictive biomarkers to select the patients for specific combination therapy and monitoring the response to ICIs therapy. The use of predictive biomarkers can bring a longdesired shift in developing personalized ICIs combination therapy for PCa patients.

\section{References}

1. Ferlay J, Ervik M, Lam F (2018) Global cancer observatory: cancer today. International Agency for Research.

2. Prostate Cancer Side Effects: Prostate Cancer Foundation.

3. Shelley M, Harrison C, Coles B, Staffurth J, Wilt TJ, et al. (2006) Chemotherapy for hormone-refractory prostate cancer. Cochrane database Syst Rev 4: 005247.

4. Petrylak DP, Tangen CM, Hussain MHA, Lara PNJ, Jones JA, et al. (2004) Docetaxel and estramustine compared with mitoxantrone and prednisone for advanced refractory prostate cancer. N Engl J Med 351(15): 1513-1520.

5. de Bono JS, Logothetis CJ, Molina A, Fizazi K, North S, et al. (2011) Abiraterone and increased survival in metastatic prostate cancer. N Engl J Med 364(21): 1995-2005.

6. Scher HI, Fizazi K, Saad F, Taplin ME, Sternberg CN, et al. (2012) Increased survival with enzalutamide in prostate cancer after chemotherapy. N Engl J Med 367(13): 11871197.

7. Parker C, Nilsson S, Heinrich D, Helle SI, O Sullivan JM, et al. (2013) Alpha emitter radium-223 and survival in metastatic prostate cancer. N Engl J Med 369: 213-223.

8. Patel SA, Hoffman Censits J (2017) Cabazitaxel in the treatment of metastatic castration-resistant prostate cancer: patient selection and special considerations. Onco Targets Ther 10: 4089-4098.

9. Kantoff PW, Higano CS, Shore ND, Berger ER, Small EJ, et al. (2010) Sipuleucel-T immunotherapy for castrationresistant prostate cancer. N Engl J Med 363(5): 411-422.

10. Moreira DM, Howard LE, Sourbeer KN, Amarasekara HS, Chow LC, et al. (2017) Predicting time from metastasis to overall survival in castration-resistant prostate cancer: 
results from SEARCH. Clin Genitourin Cancer 15(1): 6066.

11. Beer TM, Armstrong AJ, Rathkopf DE, Loriot Y, Sternberg $\mathrm{CN}$, et al. (2014) Enzalutamide in metastatic prostate cancer before chemotherapy. N Engl J Med 371(5): 424433.

12. Graff JN, Alumkal JJ, Drake CG, Thomas GV, Redmond WL, et al. (2016) Early evidence of anti-PD-1 activity in enzalutamide-resistant prostate cancer. Oncotarget 7(33): 52810-52817.

13. Hansen A, Massard C, Ott PA, Haas N, Lopez J, et al. (2016) Pembrolizumab for patients with advanced prostate adenocarcinoma: Preliminary results from the KEYNOTE-028 study. Annals of Oncology 27(6): 243265.

14. Wei SC, Duffy CR, Allison JP (2018) Fundamental mechanisms of immune checkpoint blockade therapy. Cancer Discov 8(9): 1069-1086.

15. Rittmeyer A, Barlesi F, Waterkamp D, Park K, Ciardiello F, et al. (2017) Atezolizumab versus docetaxel in patients with previously treated non-small-cell lung cancer (OAK): a phase 3 , open-label, multicentre randomised controlled trial. Lancet 389(10066): 255-265.

16. Topalian SL, Hodi FS, Brahmer JR, Gettinger SN, Smith DC, et al. (2012) Safety, activity, and immune correlates of anti-PD-1 antibody in cancer. N Engl J Med 366(26): $2443-2454$.

17. Bracarda S, Altavilla A, Hamzaj A, Sisani M, Marrocolo F, et al. (2015) Immunologic checkpoints blockade in renal cell, prostate, and urothelial malignancies. Semin Oncol 42(3): 495-505.

18. Kwon ED, Drake CG, Scher HI, Fizazi K, Bossi A, et al. (2014) Ipilimumab versus placebo after radiotherapy in patients with metastatic castration-resistant prostate cancer that had progressed after docetaxel chemotherapy (CA184-043): a multicentre, randomised, doubleblind, phase 3 trial. Lancet Oncol 15(7): 700-712.

19. Hodi FS, O'Day SJ, McDermott DF, Weber RW, Sosman JA, et al. (2010) Improved survival with ipilimumab in patients with metastatic melanoma. The New England journal of medicine 363(8): 711-723.

20. Beer TM, Kwon ED, Drake CG, Fizazi K, Logothetis C, et al. (2017) Randomized, double-blind, Phase III trial of ipilimumab versus placebo in asymptomatic or minimally symptomatic patients with metastatic chemotherapy-naïve castrationresistant prostate cancer.
J Clin Oncol 35(1): 40-47.

21. Brahmer JR, Drake CG, Wollner I, Powderly JD, Picus J, et al. (2010) Phase I study of single-agent anti-programmed death-1 (MDX-1106) in refractory solid tumors: safety, clinical activity, pharmacodynamics, and immunologic correlates. J Clin Oncol 28(19): 3167-3175.

22. Antonarakis ES, Piulats JM, Gross-Goupil M, Goh J, Ojamaa K, et al. (2020) Pembrolizumab for treatmentrefractory metastatic castration-resistant prostate cancer: multicohort, open-label Phase II KEYNOTE-199 study. J Clin Oncol 38(5): 395-405.

23. Hansen AR, Massard C, Ott PA, Haas NB, Lopez JS, et al. (2018) Pembrolizumab for advanced prostate adenocarcinoma: findings of the KEYNOTE-028 study. Ann Oncol 29(8): 1807-1813.

24. Kim JW, Shaffer DR, Massard C, Powles T, Harshman LC, et al. (2018) A phase Ia study of safety and clinical activity of atezolizumab (atezo) in patients (pts) with metastatic castrationresistant prostate cancer (mCRPC). J Clin Oncol 36(6): 187.

25. Li D, Chen R, Wang YW, Fornace AJ Jr, Li HH (2017) Prior irradiation results in elevated programmed cell death protein 1 (PD-1) in T cells. Int J Radiat Biol 94(5): 488494.

26. Bishop JL, Sio A, Angeles A, Roberts ME, Azad AA, et al. (2015) PD-L1 is highly expressed in Enzalutamide resistant prostate cancer. Oncotarget 6(1): 234-242.

27. Graff JN, Alumkal JJ, Drake CG, Thomas GV, Redmond WL, et al. (2016) First evidence of significant clinical activity of PD-1 inhibitors in metastatic, castration resistant prostate cancer (mCRPC). Annals of Oncology 27(6): 243-265.

28. Dudzinski OS, Cameron DB, Wang J, Rathmell CJ, Giorgio DT, et al. (2019) Combination immunotherapy and radiotherapy causes an abscopal treatment response in a mouse model of castration resistant prostate cancer. J Immunother Cancer 7(1): 218-225.

29. Gubin MM, Schreiber RD (2015) CANCER. The odds of immunotherapy success. Science 350(6257):158-159.

30. Robert C, Long GV, Brady B, Dutriaux C, Maio M, et al. (2015) Nivolumab in previously untreated melanoma without BRAF mutation. N Engl J Med 372(4): 320-330.

31. Brahmer J, Reckamp KL, Baas P, Crino L, Eberhardt WE, et al. (2015) Nivolumab versus Docetaxel in Advanced Squamous-Cell Non-Small-Cell Lung Cancer. N Engl J Med 373: 123-135. 
32. Borghaei H, Horn L, Spigel DR, Steins M, Ready NE, et al. (2015) Nivolumab versus Docetaxel in Advanced Nonsquamous Non-Small-Cell Lung Cancer. N Engl J Med 373(17): 1627-1639.

33. Vogelstein B, Papadopoulos N, Velculescu VE, Zhou S, Diaz LA, et al. (2013) Cancer genome landscapes. Science 339(6127): 1546-1558.

34. Robinson D, Van Allen EM, Wu YM, Schultz N, Lonigro RJ, et al. (2015) Integrative Clinical Genomics of Advanced Prostate Cancer. Cell 161(5): 1215-1228.

35. Strickland KC, Howitt BE, Shukla SA, Rodig S, Ritterhouse LL, et al. (2016) Association and prognostic significance of BRCA1/2-mutation status with neoantigen load, number of tumor-infiltrating lymphocytes and expression of PD-1/PD-L1 in high grade serous ovarian cancer. Oncotarget 7(12): 13587-13598.

36. Pritchard CC, Mateo J, Walsh MF, De Sarkar N, Abida W, et al. (2016) Inherited DNA-Repair Gene Mutations in Men with Metastatic Prostate Cancer. N Engl J Med 375(5): 443-453.

37. Pritchard CC, Morrissey C, Kumar A, Zhang X, Smith C, et al. (2014) Complex MSH2 and MSH6 mutations in hypermutated microsatellite unstable advanced prostate cancer. Nat Commun 5: 4988-4993.

38. Rodrigues DN, Rescigno P, Liu D, Yuan W, Carreira $S$, et al. (2018) Immunogenomic analyses associate immunological alterations with mismatch re-pair defects in prostate cancer. J Clin Invest 128(10): 4441-4453.

39. Wu YM, Cieslik M, Lonigro RJ, Vats P, Reimers MA, et al. (2018) Inactivation of CDK12 delineates a distinct immunogenic class of advanced prostate cancer. Cell 173(7): 1770-1782.

40. (2017) Library Meeting, Combination of PDL-1 and PARP inhibition in an unselected population with metastatic castrate-resistant prostate cancer (mCRPC).

41. Abida, W, Cheng ML, Armenia J, Middha S, Autio KA, et al. (2019) Analysis of the prevalence of microsatellite instability in prostate cancer and response to immune checkpoint blockade. JAMA Oncol 5(4): 471-478.

42. Michael AC, Brahmer JR (2018) Overcoming Resistances to immunotherapy-requires-advanced-strategies. Targeted Oncology.

43. Jayaprakash P, Ai M, Liu A, Budhani P, Bartkowiak $\mathrm{T}$, et al. (2018) Targeted hypoxia reduction restores $\mathrm{T}$ cell infiltration and sensitizes prostate cancer to immunotherapy. J Clin Invest 128(11): 5137-5149.
44. Thomas DA, Massague J (2005) TGF- $ß$ directly targets cytotoxic $\mathrm{T}$ cell functions during tumor evasion of immune surveillance. Cancer Cell 8(5): 369-380.

45. Lee J, Lee K, Kim D, Heo DS (2004) Elevated TGF-beta1 secretion and down-modulation of NKG2D underlies impaired NK cytotoxicity in cancer patients. J Immunol 172(12): 7335-7340.

46. Mariathasan S, Turley SJ, Nickles D, Castiglioni A, Yuen K, et al. (2018) TGF $\beta$ attenuates tumour response to PD-L1 blockade by contributing to exclusion of T cells. Nature 554(7693): 544-548.

47. Miller AM, Lundberg K, Ozenci V, Banham HA, Hellstrom $M$, et al. (2006) CD4+CD25high T cells are enriched in the tumor and peripheral blood of prostate cancer patients. J Immunol 177(10): 7398-7405.

48. Kiniwa Y, Miyahara Y, Wang HY, Peng W, Peng G, et al. (2007) CD8+ Foxp3+ regulatory $\mathrm{T}$ cells mediate immunosuppression in prostate cancer. Clin Cancer Res 13(23): 6947-6958.

49. Curiel TJ, Coukos G, Zou L, Alvarez X, Cheng P, et al. (2004) Specific recruitment of regulatory $T$ cells in ovarian carcinoma fosters immune privilege and predicts reduced survival. Nat Med 10(9): 942-949.

50. Nishikawa H, Sakaguchi S (2010) Regulatory T cells in tumor immunity. Int J Cancer 127(4): 759-767.

51. Obermajer N, Muthuswamy R, Odunsi K, Edwards RP, Kalinski P (2011) PGE(2)-induced CXCL12 production and CXCR4 expression controls the accumulation of human MDSCs in ovarian cancer environment. Cancer Res 71(24): 7463-7470.

52. Zou L, Barnett B, Safah H, Larussa VF, Hogan EM, et al. (2004) Bone marrow is a reservoir for CD4p CD25p regulatory $\mathrm{T}$ cells that traffic through CXCL12/CXCR4 signals. Cancer Res 64(22): 8451-8455.

53. Zou W, Machelon V, L'Hermin CA, Borvak J, Nome F, et al. (2001) Stromal-derived factor-1 in human tumors recruits and alters the function of plasmacytoid precursor dendritic cells. Nature Medicine 7(12): 13391346.

54. Demaria S, Golden EB, Formenti SC (2015) Role of local radiation therapy in cancer immunotherapy. JAMA Oncol 1(9): 1325-1332.

55. Kaur P, Asea A (2012) Radiation-induced effects and the immune system in cancer. Front Oncol 2: 191-200.

56. Postow MA, Callahan MK, Barker CA, Yamada Y, Yuan J, et 
al. (2012) Immunologic correlates of the abscopal effect in a patient with melanoma. N Engl J Med 366(10): 925931.

57. Dewan MZ, Galloway AE, Kawashima N, Dewyngaert JK, Babba JS, et al. (2009) Fractionated but not single-dose radiotherapy induces an immune-mediated abscopal effect when combined with anti-CTLA-4 antibody. Clin Cancer Res 15(17): 5379-5388.

58. Schaue D, Ratikan JA, Iwamoto KS (2012) Maximizing tumor immunity with fractionated radiation. Int J Radiat Oncol Biol Phys 83(4): 1306-1310.

59. Bang A, Wilhite TJ, Pike LRG, Cagney DN, Aizer AA, et al. (2017) Multicenter evaluation of the tolerability of combined treatment with PD-1 and CTLA-4 immune checkpoint inhibitors and palliative radiation therapy. Int J Radiat Oncol Biol Phys 98(2): 344-351.

60. Welsh JW, Tang C, de Groot P, Naing A, Hess KR, et al. (2019) Phase II trial of ipilimumab with stereotactic radiation therapy for metastatic disease: outcomes, toxicities, and low-dose radiation-related abscopal responses. Cancer Immunol Res 7(12): 1903-1909.

61. Horn L, Mansfield AS, Szczęsna A, Havel L, krzakowski $\mathrm{M}$, et al. (2018) IMpower133 Study Group. First-line atezolizumab plus chemotherapy in extensive-stage small-cell lung cancer. N Engl J Med 379(23): 2220-2229.

62. Schmid P, Adams S, Rugo HS, schneeweiss A, Barrios $\mathrm{CH}$, et al. (2018) IMpassion130 Trial Investigators. Atezolizumab and nab-paclitaxel in advanced triplenegative breast cancer. N Engl J Med 379(22): 21082121.

63. West H, McCleod M, Hussein M, Morabito A, Ritmeyer A, et al. (2019) Atezolizumab in combination with carboplatin plus nab-paclitaxel chemotherapy compared with chemotherapy alone as first-line treatment for metastatic non-squamous non-small-cell lung cancer (IMpower130): a multicentre, randomised, open-label, phase 3 trial. Lancet Oncol 20(7): 924-937.

64. Gandhi L, Rodríguez Abreu D, Gadgeel S, Esteban E, Felip E, et al. (2018) Pembrolizumab plus chemotherapy in metastatic non-small-cell lung cancer. N Engl J Med 378(22): 2078-2092.

65. Dosset $M$, Vargas TR, Lagrange A, Boidot R, Vegran F, et al. (2018) PD-1/PD-L1 pathway: an adaptive immune resistance mechanism to immunogenic chemotherapy in colorectal cancer. Oncoimmunology 7(6): e1433981.

66. Alizadeh D, Trad M, Hanke NT, Larmonier CB, Janikashvili
$\mathrm{N}$, et al. (2014) Doxorubicin eliminates myeloid-derived suppressor cells and enhances the efficacy of adoptive T-cell transfer in breast cancer. Cancer Res 74(1): 104118.

67. Lasko LM, Jakob CG, Edalji RP, Qiu W, Montgomery D, et al. (2017) Discovery of a selective catalytic p300/ CBP inhibitor that targets lineage-specific tumours. Nature 550(7674): 128-132.

68. Asangani IA, Dommeti VL, Wang X, Malik R, Cieslik M, et al. (2014) Therapeutic targeting of BET bromodomain proteins in castration-resistant prostate cancer. Nature 510(7504): 278-282.

69. Yang YA, Yu J (2013) EZH2, an epigenetic driver of prostate cancer. Protein Cell 4(5): 331-341.

70. Liu J, He D, Cheng L, Huang C, Zhang Y, et al. (2020) p300/ CBP inhibition enhances the efficacy of programmed death-ligand 1 blockade treatment in prostate cancer. Oncogene 39(19): 3939-3951.

71. Katoh M (2016) Mutation spectra of histone methyltransferases with canonical SET domains and EZH2-targeted therapy. Epigenomics 8(2): 285-305.

72. Sheahan AV, Morel KL, Burkhart DL, Baca SC, labbi DP, et al. (2019) Targeting EZH2 Increases Therapeutic Efficacy of Check-Point Blockade in Models of Prostate Cancer.

73. Peng D, Kryczek I, Nagarsheth N, Zhao L, Wei S, et al. (2015) Epigenetic silencing of TH1-type chemokines shapes tumour immunity and immunotherapy. Nature 527(7577): 249-253.

74. Ennishi D, Takata K, Beguelin W, Duns G, Mottok A, et al. (2019) Molecular and Genetic Characterization of MHC Deficiency Identifies EZH2 as Therapeutic Target for Enhancing Immune Recognition. Cancer discovery 9(4): 546-563.

75. Batlle E, Massague J (2019) Transforming growth factor- $\beta$ signaling in immunity and cancer. Immunity 50(4): 924-940.

76. Jiao S, Subudhi SK, Aparicio A, Zhongqi Ge, Baoxiang Guan, et al. (2019) Differences in tumor microenvironment dictate $\mathrm{T}$ helper lineage polarization and response to immune checkpoint therapy. Cell 179(5): 1177-1190.

77. Lopez Bujanda ZA, Haffner MC, Chaimowitz MG, Chowdhury N, Venturini NJ, et al. (2019) Castrationmediated IL-8 promotes myeloid infiltration and prostate cancer progression. bioRxiv 13: 44. 
78. Vigano S, Alatzoglou D, Irving M, Cauxet CM, Cauxal C, etal. (2019) Targeting Adenosine in Cancer Immunotherapy to Enhance T-Cell Function. Front Immunol 10: 925.

79. Bendell J, Bauer T, Patel M, Falchook G, Karlixet JL, et al. (2019) Abstract CT026: Evidence of immune activation in the first-in-human phase Ia dose escalation study of the adenosine 2a receptor antagonist, AZD4635, in patients with advanced solid tumors. Cancer Research 79(13).

80. Gamat M, McNeel DG (2017) Androgen deprivation and immunotherapy for the treatment of prostate cancer. Endocr Relat Cancer 24(12): T297-T310.

81. Mercader M, Bodner BK, Moser MT, Kwon PS, Park ES, et al. (2001) T cell infiltration of the prostate induced by androgen withdrawal in patients with prostate cancer. Proc Natl Acad Sci USA 98(25): 14565-14570.

82. Li A, Yi M, Qin S, Chu Q, Luo S, et al. (2019) Prospects for combining immune checkpoint blockade with PARP inhibition. J Hematol Oncol 12(1): 98.

83. Stewart RA, Pilie PG, Yap TA (2018) Development of PARP and immune-checkpoint inhibitor combinations. Cancer Res 78(24): 6717-6725.

84. Jiao S, Xia W, Yamaguchi H, Wei Y, Chen MK, et al. (2017) PARP inhibitor Upregulates PD-L1 expression and enhances cancer-associated immunosuppression. Clin Cancer Res 23(14): 3711-3720.

85. Karzai F, Madan RA, Owens H, Couvillon A, Hankin A, et al. (2018) A phase 2 study of olaparib and durvalumab in metastatic castrate-resistant prostate cancer (mCRPC) in an unselected population. J Clin Oncol 36(S6): 163.

86. Karzai F, Vander Weele D, Madan RA, Owens H, Cordes LM, et al. (2018) Activity of durvalumab plus olaparib in metastatic castration-resistant prostate cancer in men with and without DNA damage repair mutations. J Immunother Cancer 6(1): 141-152.

87. Ott PA, Bang YJ, Piha Paul SA, Abdul Razak AR, Bennouna J, et al. (2019) T-cell-inflamed gene-expression profile, programmed death ligand 1 expression, and tumor mutational burden predict efficacy in patients treated with pembrolizumab across 20 cancers: KEYNOTE-028. J Clin Oncol 37:3 18-327.

88. Hansen AR, Massard C, Ott PA, Haas NB, Lopez JS, et al. (2018) Pembrolizumab for advanced prostate adenocarcinoma: findings of the KEYNOTE-028 study. Ann Oncol 29(8): 1807-1813.

89. Antonarakis ES, Piulats JM, Gross Goupil M, Goh J,
Ojamaa K, et al. (2020) Pembrolizumab for treatmentrefractory metastatic castration-resistant prostate cancer: Multicohort, open-label phase II KEYNOTE-199 study. J Clin Oncol 38(5): 395-405.

90. Nishino M, Ramaiya NH, Hatabu H, Hodi FS (2017) Monitoring immune-checkpoint blockade: response evaluation and biomarker development. Nat Rev Clin Oncol 14(11): 655-668.

91. Topalian SL, Taube JM, Anders RA, Pardoll DM (2016) Mechanism-driven biomarkers to guide immune checkpoint blockade in cancer therapy. Nat Rev Cancer 16: $275-287$.

92. Lu S, Stein JE, Rimm DL, Wang DW, Bell JM, et al. (2019) Comparison of biomarker modalities for predicting response to PD-1/PD-L1 checkpoint blockade. JAMA Oncol 5(8): 1195-1204.

93. Davis AA, Patel VG (2019) The role of PD-L1 expression as a predictive biomarker: an analysis of all U.S. Food and Drug Administration (FDA) approvals of immune checkpoint inhibitors. J Immunother Cancer 7(1): 278.

94. McLaughlin J, Han G, Schalper KA, Hausdorf CD, Pelekanou V, et al. (2016) Quantitative assessment of the heterogeneity of PD-L1 expression in non-small-cell lung cancer. JAMA Oncol 2(1): 46-54.

95. Tang Y, Zhang P, Wang Y, Wang J, Su M, et al. (2020) The Biogenesis, Biology, and Clinical Significance of Exosomal PD-L1 in Cancer. Front Immunol 11: 604.

96. Chen G, Huang AC, Zhang W, Zhang G, Wu M, et al. (2018) Exosomal PD-L1 contributes to immunosuppression and is associated with anti-PD-1 response. Nature 560(7718): 382-386.

97. Poggio M, Hu T, Pai CC, Chu B, Belair CD, et al. (2019) Suppression of exosomal PD-L1 induces systemic antitumor immunity and memory. Cell 177(2): 414-427.

98. Del Re M, Marconcini R, Pasquini G, Rofi E, Vivaldi C, et al. (2018) PD-L1 mRNA expression in plasma-derived exosomes is associated with response to anti-PD-1 antibodies in melanoma and NSCLC. Br J Cancer 118(6): 820-824.

99. Chalmers ZR, Connelly CF, Favrizio D, Gay L, Ali SM, et al. (2017) Analysis of 100,000 human cancer genomes reveals the landscape of tumor mutational burden. Genome Med 9(1): 34.

100. Rizvi NA, Hellmann MD, Snyder A, Kvistborg P, Makarov V, et al. (2015) Cancer immunology. Mutational landscape determines sensitivity to PD-1 blockade in non-small cell lung cancer. Science 
348(6230): 124-128.

101. Mehra N, van Riet J, Smits M, Westdorp M, Gorris T, et al. (2018) 798PD-In-depth assessment of metastatic prostate cancer with high tumour mutational burden. Ann Oncol 29 (S8): 271-302.

102. Antonarakis ES, Shaukat F, Isaacsson Velho P, Kaur H, Shenderov $E$, et al. (2019) Clinical features and therapeutic outcomes in men with advanced prostate cancer and DNA mismatch repair gene mutations. Eur Urol 75(3): 378-382.
103. Le DT, Uram JN, Wang H, Bartlett BR, Kemberling H, et al. (2015) PD-1 blockade in tumorswith mismatch-repair deficiency. NEngl J Med372(26): 2509-2520.

104. Sato H, Niimi A, Yasuhara T, Permata TBM, Hagiwara Y, et al. (2018) DNA double-strand break repair pathway regulates PD-L1 expression in cancer cells. Nat Commun 8: 1751.

105. LangS,SwiftS,WhiteH,MissoK,KleijnenJ,etal.(2019)Asystematic review of the prevalence ofDNAdamage responsegenemutations in prostate cancer. Int J Oncol 55(3): 597-616. 